\title{
Experimental Validation of a Dynamic Mooring Lines Code with Tension and Motion Measurements of a Submerged Chain
}

\author{
José Azcona $^{\mathrm{a}, *}$, Xabier Munduate ${ }^{\mathrm{a}}$, Leo González ${ }^{\mathrm{b}}$, Tor A Nygaard ${ }^{\mathrm{c}}$ \\ ${ }^{a}$ National Renewable Energy Centre, CENER \\ Ciudad de la Innovación 7, 31621 Sarriguren, Navarra, Spain \\ Telephone: +34948252800 \\ ${ }^{b}$ Universidad Politécnica de Madrid, UPM \\ ETSI Navales, Avd. Arco de la Victoria, 4, Ciudad Universitaria, 28040 Madrid, Spain \\ Telephone: +34913367156 \\ ${ }^{c}$ Institute for Energy Technology, IFE \\ Instituttveien 18, P.O. Box 40, NO-2007 Kjeller, Norway \\ Telephone: +4763806000
}

\begin{abstract}
This paper shows a complete study of the dynamics of a mooring line: first, a numerical simulation code based on a lumped mass formulation was developed and tested under different setups and second, an equivalent experimental campaign was performed to compare against the numerical predictions. The tests consisted of a suspended chain submerged into a water basin, where the suspension point of the chain was excited with harmonic motions of different periods. The code is able to predict the tension at the suspension point and the motions at several positions of the line with high accuracy. Even for those cases where the line loses and subsequently recovers tension, the resulting snap load and motions are well captured with a slight overprediction of the maximum tension. The drag coefficients for chains used in the computations have been taken from the DNV guidelines and in general predict correctly the hydrodynamic loads. In addition, sensitivity studies and verification against another code show that highly dynamic cases are sensitive to the seabed-cable contact and friction models. The results show the importance of capturing the evolution of the mooring
\end{abstract}

*Correspondence: jazcona@cener.com 
dynamics for the prediction of the line tension, especially for the high frequency motions.

Keywords: mooring line, lumped mass, dynamic, experiment, validation, wave tank

2010 MSC: 00-01, 99-00

\section{Introduction}

The mooring system of an offshore floating structure consists of several lines attached to the structure by fairleads with their corresponding lower ends anchored to the seabed. The mooring system holds a floating structure in the

5 desired location (station keeping) and, for certain platform designs, such as Tension Leg Platforms (TLP), provides a restoring moment that contributes to counteract the overturning moment. The influence of mooring lines on the global dynamics of a floating wind turbine can be an important factor determining the system motion and the loads of the different components of the system 8]. The mooring system can also have an important influence on the natural frequencies of the platform.

Due to the importance of these mooring systems in the simulation of floating structures and the necessity of an accurate description of their behavior, a new numerical tool, known as OPASS (Offshore Platform Anchorage System Simulator), was developed by CENER (the Spanish National Renewable Energy Centre) for the simulation of non-linear mooring dynamics. CENER and IFE (Institute for Energy Technology, in Norway) have been collaborating during the last years in the development of dynamic mooring lines models and in the experimental validation of models with scaled tests. A first verification of the OPASS code against computations of IFE's 3DFloat code [3] was successfully accomplished in [1]. Afterwards, a verification of OPASS coupled with the well reputed code FAST was satisfactorily carried out within the IEA Annex 30 benchmark (OC4) [15]. Finally, an experimental validation has been completed against test data of a submerged chain generated at the Ècole Centrale 
de Nantes (ECN) wave tank in France. This paper describes the series of tests conducted at ECN by CENER and IFE and the results of the validation, comparing computations with experimental measurements for the chain suspension point tension, and for the motions of the line at different positions. For a highly dynamic case, the effect of seabed friction and damping was studied in more 30 detail, also by verification against the 3 DFloat code.

\section{Mooring Lines Modeling Approaches}

The equations of motion of a submerged line are non-linear and cannot be solved analytically, consequently numerical methods have to be applied. Some modeling approaches are simplified methods as the Quasi-Static or the forcedisplacement relationship. The Quasi-Static approach consists in the resolution of the static equations of the catenary at every time step of the simulation, given the position of the line-platform attachment. This method neglects the inertial effects and also the hydrodynamic drag produced by waves, currents or the movements of the line. In the force-displacement relationship model, non-linear

40 spring stiffnesses are applied to the translational and rotational degrees of freedom of the platform point where the line is connected. The force-displacement relationship has to be derived using a mooring line analysis code and the results obtained are similar to the Quasi-Static approach. Dynamic models consider effects as inertia, added mass or hydrodynamic drag, but they require higher computational effort. Several numerical formulations exist that can solve the dynamics of the line as the Finite Elements Method (FEM), the Finite Difference Method or Multi-Body models. The Lumped Mass model can be considered a variation of the FEM approach, where the mass of the elements is concentrated in the element adjacent nodes. The OPASS code, that is validated in this paper 50 is a Lumped Mass model.

The Lumped Mass method was first used to model a mooring line at the end of the 50's [19], though the model was simple and neglected the elasticity of the material, the hydrodynamic drag forces or the seabed-line contact and the 
model was not validated. This model was improved at the beginning of the 80 's including elasticity, cable-seabed interaction and drag due to the relative motion of the cable with respect to the water and was validated with forced harmonic oscillation tests in [20] and [10]. The agreement with computational models was good, though the tests only covered a limited set of cases that did not consider slack conditions. The methodology to perform the experimental tests was based in the work described a few years before in [13]. Another comparison between a computational Lumped Mass model and a submerged line with prescribed harmonic displacements at the suspension point is described in [18]. This reference shows a good agreement at the line end tension between the experimental results and the calculation, but comparisons of the cases including 65 slack condition are not shown. In [17] the experimental results of the different line configurations are divided into four different states. In the Quasi-Static Condition the frequency of the excitation is low and the dynamic effects are negligible. The Harmonic Condition appears at a higher frequency and presents a tension signal in time domain that is close to sinusoidal. In the Snap Condition, the tension drops to zero when the fairlead moves towards the anchor. When the motion reverses, the tension is recovered, and the zero loaded chain links that are falling suddenly stop and a snap loading is produced. The fourth condition appears when the excitation frequency increases even more and the upper end of the chain completes a period before the whole chain is able to respond. In this case, a lower number of chain links loose tension and the snap load amplitudes start decreasing again. In the same reference, a Lumped Mass model is compared with tension experimental results with good agreement for the Harmonic condition, but presents significant differences when the line loses tension. The importance in the mooring lines dynamic modeling of the accurate identification of the cable elastic stiffness and the free falling velocity is highlighted in [12]. This work also presents a comparison between the experimentally measured tension and numerical computations showing good agreement. In [16], dynamic simulations with Orcaflex [11] are compared to experimental data of a mooring line with prescribed harmonic motion of the fairlead. The experiments 
include cases with and without current. Good agreement between tension in computations and experiments is achieved except for the cases where the line loses tension. The computed results here show spikes of much higher tension than in the experiments. More recently, a Lumped Mass mooring line code has been validated against tension measurements of a floating wind turbine mooring lines obtained with scaled tests in a wave tank [5].

\section{Characteristics of the OPASS Code}

OPASS is a dynamic code based in Finite Elements Method with three translational degrees of freedom defined at each node and the element mass lumped at the nodes. The code model consists of an slender line with constant circular section. It considers the effect of inertia, hydrodynamic added mass, gravity, hydrostatics, wave kinematics, tangential and normal hydrodynamic drag, axial elasticity and structural damping. The code neglects the bending stiffness, therefore, it is suitable for the simulation of chains. The contact of the line with the seabed is also included. The code can compute as an stand-alone tool for the simulation of one mooring line or coupled with the FAST code [7], whose original mooring lines model is a Quasi-Static approach, for the integrated simulation of floating offshore wind turbines.

\section{Basic Dynamic Equations}

A mooring line has one of his ends fixed to the seabed by an anchor and the upper end is attached to the floating platform, usually using a fairlead. Part of the line can be in contact with the seabed. A coordinate system $l_{0}$ is defined in the cable as the distance along the unstretched length of the cable, from the anchor to the cable section to be considered.

The cable is a very slender structure and consequently shear forces can be neglected. If bending and torsion stiffness are low enough to also be neglected, 
the only internal forces are the tension $T$ and the structural damping $F_{D}$, being both internal forces always tangential to the cable. The external forces acting on the cable are gravity, buoyancy and the hydrodynamic drag force. There is also an additional inertial force due to the volume of water displaced by the moving line (added mass).

Figure 1 shows the forces acting on an infinitesimal element of cable $d l$, at point $P$, that is located at a distance $l_{0}$ along the unstretched length of the cable. The resultant force from hydrostatic pressure and gravity, $\vec{F}_{1}$, is vertical. The hydrodynamic drag force is split in two components: $\vec{F}_{2}$ and $\vec{F}_{3}$, normal and tangential to the cable respectively. The inertial force coming from the added mass, $\vec{F}_{4}$, is supposed to have only a component normal to the cable. All these forces are expressed in the global reference system per unit of unstretched length. According to Figure 1, and including the inertial forces in the general

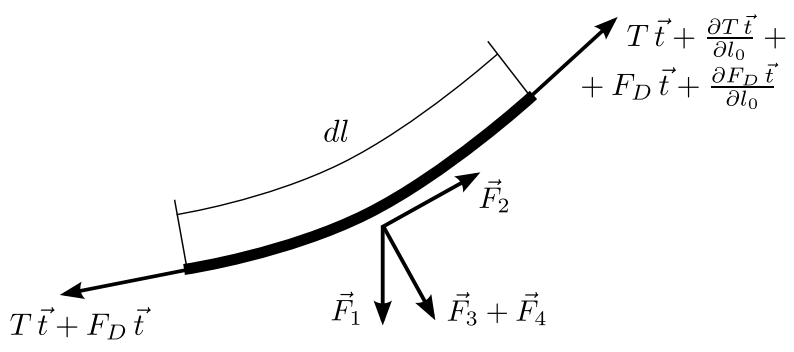

Figure 1: Forces acting on an infinitesimal length of cable

balance, the equation that represents the dynamics of the mooring line in the global coordinate system results:

$$
\gamma \overrightarrow{\ddot{R}}-\frac{\partial(T \vec{t})}{\partial l_{0}}-\frac{\partial\left(F_{D} \vec{t}\right)}{\partial l_{0}}-\left(\vec{F}_{1}+\vec{F}_{2}+\vec{F}_{3}+\vec{F}_{4}\right)=0
$$

Where $\gamma$ is the line mass per unit of cable unstretched length, $\vec{R}$ is the acceleration of the point $P$ and $\vec{t}$ is the vector tangential to the cable at point $P$ in the global reference system.

If we relate the displacements of the cable to an initial reference cable configuration $R$, then the current position vector $\vec{R}$ of point $P$ can be expressed in the 
global reference system as:

$$
\vec{R}=\vec{R}_{0}+\vec{U}
$$

Where $\vec{R}_{0}$ is the initial position vector of point $P$ at the reference line configuration $R$, and $\vec{U}$ is the displacement vector.

If $\vec{R}$ is the position vector of point $P$ in the global system, the vector tangential to the line at point $P$ in equation (11) can be calculated as:

$$
\vec{t}=\frac{\frac{\partial \vec{R}}{\partial l_{0}}}{\left|\frac{\partial \vec{R}}{\partial l_{0}}\right|}
$$

The axial deformation of the considered element, $\varepsilon$, is defined as:

$$
\varepsilon=\frac{\partial l}{\partial l_{0}}-1=\left|\frac{\partial \vec{R}}{\partial l_{0}}\right|-1,
$$

Where $l$ is the distance along the stretched length of the cable.

The tension at point $P$ is obtained from the constitutive equation:

$$
T=E A \varepsilon,
$$

Where $E$ is the material Young's modulus and $A$ is the section area of the cable. The calculation of the structural damping force, $F_{D}$, is based on the Rayleigh model and is proportional to the stiffness $E A$ with a proportionality coefficient $\beta$ :

$$
F_{D}=\beta E A \dot{\varepsilon},
$$

Where $\dot{\varepsilon}$ is the deformation velocity.

The resultant force from buoyancy and gravity per unit of unstretched length, expressed by the vertical force $\vec{F}_{1}$, is the weight of the cable minus the weight of the displaced volume of water per unit of unstretched length. Thus:

$$
\vec{F}_{1}=\left[\begin{array}{c}
0 \\
0 \\
-\gamma_{r} g
\end{array}\right]
$$

and

$$
\gamma_{r}=\frac{\rho_{c}-\rho_{w}}{\rho_{c}} \gamma
$$


Where $g$ is the gravity constant, $\gamma_{r}$ is the equivalent mass per unit length of the cable submerged in water, $\rho_{c}$ is the density of the cable and $\rho_{w}$ is the density of the water.

The hydrodynamic forces considered in Equation (1) are the tangential drag component $\vec{F}_{2}$, the normal drag component $\vec{F}_{3}$ and the hydrodynamic inertial force $\vec{F}_{4}$, which is also normal to the cable. These hydrodynamic forces are represented in Figure 2 ,

Using the Morison equation for slender cylinders 9 , the value of the normal

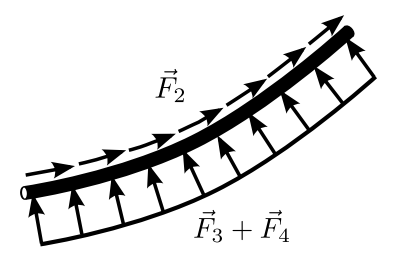

Figure 2: Tangential drag, normal drag and added mass force along the cable

drag force per unit length of the unstretched cable can be calculated as:

$$
\begin{gathered}
\vec{F}_{3}=C_{3}\left|\vec{V}_{n}\right| \vec{V}_{n}(1+\varepsilon)=C_{3}\left[\vec{V} \cdot \vec{V}-(\vec{V} \cdot \vec{t})^{2}\right]^{\frac{1}{2}}[\vec{V}-(\vec{V} \cdot \vec{t}) \vec{t}](1+\varepsilon) \\
C_{3}=\frac{1}{2} C_{d n} D \rho_{w},
\end{gathered}
$$

Where $C_{d n}$ is the normal drag coefficient and $\vec{V}_{n}$ is the normal component of the relative velocity between the water and the cable.

A similar equation can be used to calculate the drag force tangential to the cable per unit length:

$$
\begin{gathered}
\vec{F}_{2}=C_{2}\left|\vec{V}_{t}\right| \vec{V}_{t}(1+\varepsilon)=C_{2}(|\vec{V} \cdot \vec{t}| \vec{V} \cdot \vec{t})(1+\varepsilon) \vec{t} \\
C_{2}=\frac{1}{2} C_{d t} D \rho_{w}
\end{gathered}
$$

Where $C_{d t}$ is the tangential drag coefficient and $D$ is the diameter of the cable. The vector $\vec{V}$ denotes the relative velocity between the water and the cable and $\vec{V}_{t}$ is the component tangential to the element, both expressed in the global reference system. 
The hydrodynamic inertial force per unit of unstretched cable length is:

$$
\begin{gathered}
\vec{F}_{4}=-C_{4}(1+\varepsilon)[\vec{R}-(\overrightarrow{\ddot{R}} \cdot \vec{t}) \vec{t}] \\
C_{4}=C_{m n} \frac{\pi D^{2}}{4} \rho_{w}
\end{gathered}
$$

Where $C_{m n}$ is the normal added mass coefficient.

Finally, substituting equations (5), (6) and (11) into (10) results in:

$\gamma \overrightarrow{\ddot{R}}+C_{4}(1+\varepsilon)[\overrightarrow{\ddot{R}}-(\overrightarrow{\ddot{R}} \cdot \vec{t}) \vec{t}]-\frac{\partial(E A \varepsilon \vec{t})}{\partial l_{0}}-\frac{\partial(\beta E A \dot{\varepsilon} \vec{t})}{\partial l_{0}}-\vec{F}_{1}-\vec{F}_{2}-\vec{F}_{3}=0$

The Virtual Works Principle is applied multiplying Equation (12) by a virtual displacement $\delta \vec{U}$, integrating along the length of the line $L$ and equaling to 0 .

$$
\begin{aligned}
& W_{V}=\int_{0}^{L}\left\{\left(\gamma \vec{R}+C_{4}(1+\varepsilon)[\vec{R}-(\overrightarrow{\ddot{R}} \cdot \vec{t}) \vec{t}]\right) \cdot \delta \vec{U}-\left(\vec{F}_{1}+\vec{F}_{2}+\vec{F}_{3}\right) \cdot \delta \vec{U}+\right. \\
& \left.+E A \varepsilon \frac{\partial \delta \vec{U}}{\partial l_{0}} \cdot \vec{t}+\beta E A \dot{\varepsilon} \frac{\partial \delta \vec{U}}{\partial l_{0}} \cdot \vec{t}\right\} d l_{0}-[E A \varepsilon \vec{t} \cdot \delta \vec{U}]_{0}^{L}-[\beta E A \dot{\varepsilon} \vec{t} \cdot \delta \vec{U}]_{0}^{L}=0
\end{aligned}
$$

The last two terms represent the work performed by the external forces acting at the initial and final faces of the cable. If the anchor and the suspension point of the cable are fixed or their displacements are prescribed, these terms are zero. Considering our particular boundary conditions, where the anchor remains fixed and the fairlead position is prescribed or determined by the platform displacements, these two terms are neglected.

Equation (13) contains space and time derivatives, therefore, the Finite Elements Method is applied to discretize Equation (13) and transform the Partial Derivative Equations (PDE) into a set of Ordinary Derivative Equations (ODE) with only time derivatives. A Runge-Kutta-Nyström integration scheme [6] has been applied for the time integration of the resulting equations of motion. Linear polynomials have been used for the integration of the distributed forces along the elements and transfer them to the nodes. The body mass and added mass terms have been also lumped in the nodes. This produced a global mass matrix composed by $3 \times 3$ submatrices in the diagonal, which can be easily inverted, increasing the computational speed. The development of the full system 
of equations is omitted here for the sake of brevity.

The seabed is assumed to be horizontal and the line-seabed contact model is based on bi-linear springs at the nodes. When a node contacts the seabed, a spring located at the node position introduces a vertical reaction force avoiding the node to penetrate into the seabed. A damping force is also introduced in the node by the seabed. A chain-seabed friction model has been also implemented in the tangential and the normal direction to each line element. The friction forces are defined by means of a friction coefficient in the tangential and the normal directions. The friction force is computed as the product of these coefficients by the vertical seabed reaction force.

\section{Description of the Experiments}

The dimensions of the Ècole Centrale de Nantes wave tank are 50m length, $30 \mathrm{~m}$ width and $5 \mathrm{~m}$ depth. The motion tracking system that captures the motion of the reflecting markers at the different chain positions is composed by 6 Qualisys underwater cameras, type Oqus $3+$, with 1296x1024 pixels. The lenses have $20 \mathrm{~mm}$ and $24 \mathrm{~mm}$ of focal length with an averaged residual of $2 \mathrm{~mm}$. This results in an uncertainty of the marker position of approximately $2 \mathrm{~mm}$. The load cell used to measure the tension at the fairlead is a submersible DDEN model with a range of $0-500 \mathrm{~N}$ and an accuracy of $\pm 0.25 \%$. The chain was submerged into the water basin, forming a catenary shape with the bottom end anchored to the tank floor. The suspension point was connected through a load cell to a mechanical actuator with the capability of reproducing a prescribed motion that was located at the water free surface. Figure 3 shows the configuration of the experiment. During the test, the anchor remains fixed at the bottom of the basin, at a depth of $5 \mathrm{~m}$. The fairlead, located at the water plane, is excited by the mechanical actuator with a sinusoidal prescribed horizontal motion in the plane of the catenary, around a mean position. The distance $d$ is the horizontal distance between the anchor and the mean position of the fairlead during the 
tests. Two different configurations of the mooring chain corresponding to different values of the parameter $d$ are tested: Configuration 1, where $d=19.364 \mathrm{~m}$, and Configuration 2, where $d=19.870 \mathrm{~m}$. The Figure also shows the reference system that will be used to present the results.

The steel chain selected for the tests is a DIN5685A design with a link diameter
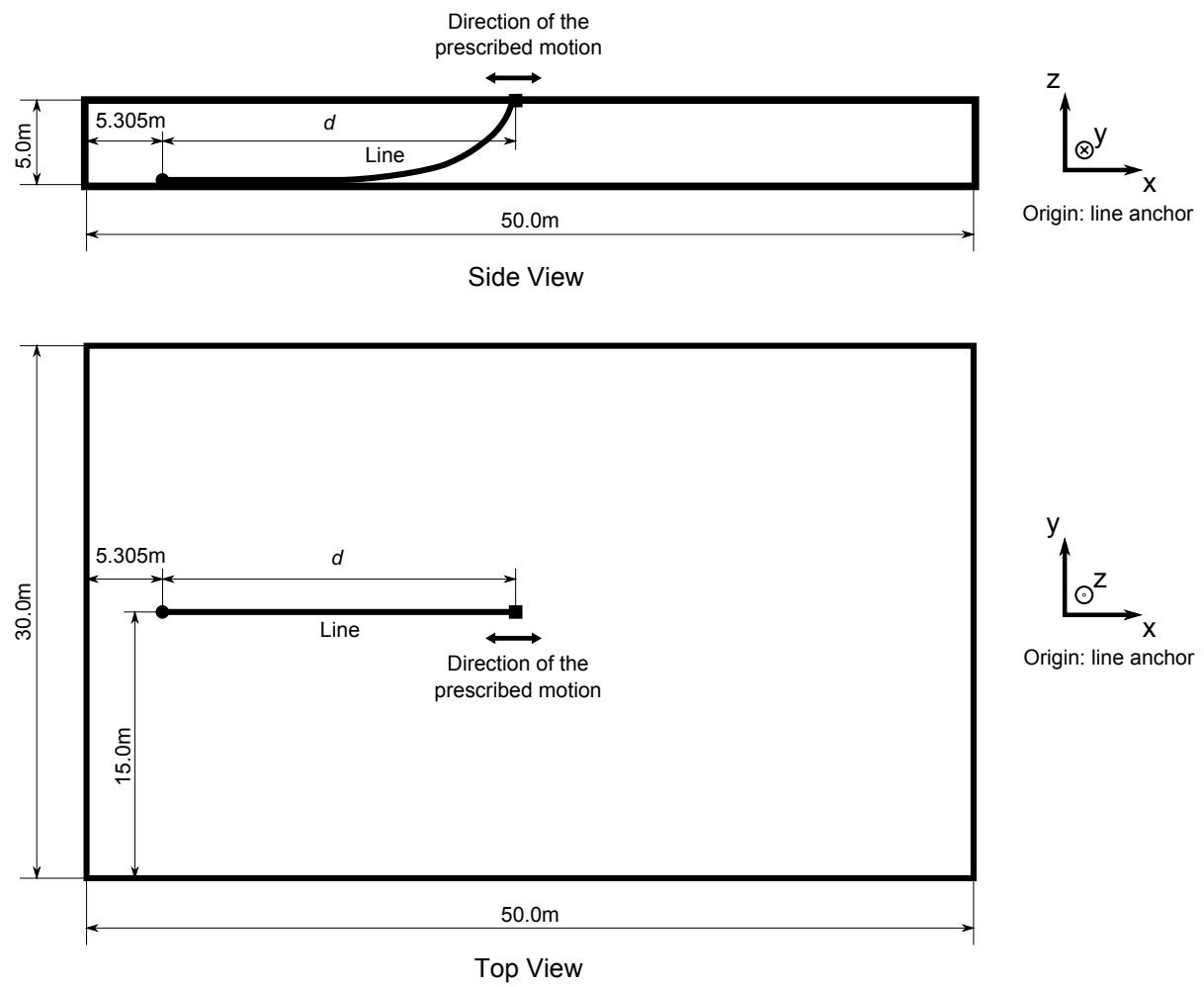

Figure 3: Configuration of the Experiment

of $2 \mathrm{~mm}$. The length of the chain including the load cell at the fairlead is $21 \mathrm{~m}$. Some preliminary simulations have been performed in order to confirm that the presence of the load cell has not an important impact on the tension and dynamics of the chain. The mass per unit length was measured and a value of $69 \mathrm{~g} / \mathrm{m}$ was found, though the original manufacturer specifications was $70 \mathrm{~g} / \mathrm{m}$. 
This chain model was chosen because the resulting full scale mass scales by an approximate factor of $1 / 40^{2}$ when compared to the properties of the OC4 floating model mooring lines [14]. With a scale factor of $1 / 40$, the $5 \mathrm{~m}$ depth of the basin represents a $200 \mathrm{~m}$ depth sea location. Therefore, this chain represents a typical scaled mooring line used in wave tank tests for floating wind turbines.

First, two static cases with the chain suspension point fixed corresponding to Configuration 1 and Configuration 2 are studied. In addition, 6 dynamic cases are simulated combining the 2 chain configurations with three different oscillation periods of the motion imposed at the fairlead. Similar criteria based on scale factors used for the chain dimensions were also used in the selection of the excitation periods of $1.58 \mathrm{~s}, 3.16 \mathrm{~s}$ and $4.74 \mathrm{~s}$. These periods correspond to oscillation periods of $10 \mathrm{~s}, 20 \mathrm{~s}$ and $30 \mathrm{~s}$ in full scale when a $1 / 40$ scale factor is used. As a matter of fact, a typical surge period of a moored platform is $10 \mathrm{~s}$ or higher. The cases that have been considered are described in Table 1.

Table 1: Description of the Test Cases

\begin{tabular}{ccccc}
\hline \hline Case & Configuration & $\begin{array}{c}\text { Anchor - Fairlead } \\
\text { Mean Distanced }(m)\end{array}$ & $\begin{array}{c}\text { Amplitude } \\
(m)\end{array}$ & $\begin{array}{c}\text { Period } \\
(s)\end{array}$ \\
\hline 1 & & 19.364 & \multicolumn{2}{c}{ Static } \\
2 & 2 & 19.872 & \multicolumn{2}{c}{ Static } \\
3 & 1 & 19.364 & 0.25 & 1.58 \\
4 & 1 & 19.364 & 0.25 & 3.16 \\
5 & 1 & 19.364 & 0.25 & 4.74 \\
6 & 2 & 19.872 & 0.25 & 1.58 \\
7 & 2 & 19.872 & 0.25 & 3.16 \\
8 & 2 & 19.872 & 0.25 & 4.74 \\
\hline
\end{tabular}

During the dynamic tests, the position of 8 reflecting markers located at different chain positions and the tension at the fairlead of the chain were measured. The distance between two adjacent reflecting markers along the chain is approxi- 
mately $0.5 \mathrm{~m}$. The exact position of these markers is provided in Table 2 .

Table 2: Position of the Markers

\begin{tabular}{cc}
\hline \hline $\begin{array}{c}\text { Marker } \\
\text { Number }\end{array}$ & $\begin{array}{c}\text { Position Alongthe Line } \\
\text { from the Fairlead }(m)\end{array}$ \\
\hline 1 & 0.656 \\
2 & 1.155 \\
3 & 1.655 \\
4 & 2.149 \\
5 & 2.646 \\
6 & 3.152 \\
7 & 3.655 \\
8 & 4.164 \\
\hline
\end{tabular}

For the static cases (cases 1 and 2), in addition to the markers in Table 2, the positions of 4 additional reflecting markers located at the lower part of the chain were also monitored. The distance between these additional reflecting markers was also $0.5 \mathrm{~m}$ approximately. The fairlead static tension was also measured.

\section{Parameters of the Computational Model}

As has been mentioned in Section 5, the measured weight per unit length of the chain is $69 \mathrm{~g} / \mathrm{m}$ and the length is $21 \mathrm{~m}$. As the computational model assumes that the chain is a line with a constant circular section, an equivalent hydrodynamic diameter has to be determined. To do this, the volume of the total length of the chain was measured and the diameter of the circular section that provides that volume for the same length was calculated assuming that the material density (steel) had a value of $7850 \mathrm{~kg} / \mathrm{m}^{3}$.

The hydrodynamic coefficients, in particular the drag coefficients, have a great influence on the mooring line dynamics, therefore a realistic selection of the values is critical to obtain accurate simulation results. An added mass coefficient, 
$C_{m n}$, of 1 was chosen according to Bureau Veritas 2]. The normal drag coefficient, $C_{d n}$, and the tangential drag coefficient, $C_{d t}$, were obtained following the indications of DNV [4]. For a studless chain, this guideline provides a value for $C_{d n}$ of 2.4 and for $C_{d t}$ of 1.15 . These values are referred to the wire diameter of the chain link. For the implementation in our code, the value has to be referred to the equivalent hydrodynamic diameter, resulting in values of 1.4 and 0.67 , respectively. The axial stiffness of the chain has been estimated based on the link diameter according to Equation (14), see [11]:

$$
E A=0.854 \cdot 10^{8} d_{w}^{2}(k N)
$$

Where $d_{w}$ is the wire diameter of the chain link.

The characteristics of the chain are summarized in Table 3

Table 3: Characteristics of the chain

\begin{tabular}{lcc}
\hline \hline & Parameters & Units \\
\hline Length & 21 & $\mathrm{~m}$ \\
Mass per unit length & 0.069 & $\mathrm{~kg} / \mathrm{m}$ \\
Density & 7850 & $\mathrm{~kg} / \mathrm{m}^{3}$ \\
Axial stiffness & $3.4 \mathrm{E} 5$ & $\mathrm{~N}$ \\
Structural damping & 0.1 & $\%$ \\
Equivalent hydrodynamic diameter & 0.0034 & $\mathrm{~m}$ \\
Added mass coefficient & 1.0 & - \\
Normal drag coefficient & 1.4 & - \\
Tangential drag coefficient & 0.67 & - \\
\hline
\end{tabular}

185

A line-seabed contact model has been defined, including the friction of the chain with the seabed. The parameters related to this model are collected in Table 4 .

A minimum of 30 elements were used to discretize the chain in the compu190 tations. A sensitivity analysis was performed running several simulations with 
Table 4: Characteristics of the line-seabed contact and friction models. The stiffness and damping values are per unit length of the line.

\begin{tabular}{lcc}
\hline \hline & Parameters & Units \\
\hline Vertical seabed stiffness & 20 & $\mathrm{~N} / \mathrm{m}^{2}$ \\
Vertical seabed damping & 0.1 & $\mathrm{Ns} / \mathrm{m}^{2}$ \\
Tangential friction coefficient & 0.5 & - \\
Normal friction coefficient & 0.5 & - \\
\hline
\end{tabular}

double number of elements with no significant changes in the results.

\section{Comparison of Computational and Experimental Results}

In this section, a comparison between the experimental results and compu-

195

tations is presented, with the objective of validating the code for the dynamic simulation of mooring lines. First, computed and experimental data of the shape and tension are presented for the static cases. Afterwards, results comparing measurements and computations of the tension at the suspension point and the positions of different chain points are shown for the dynamic cases. Results in this section are presented according to the reference system in Figure 3 ,

\subsection{Static Cases}

These cases were used to adjust the exact horizontal position of the chain anchor that was fixed to the basin bottom by a diver. In contrast to other parameters of the test that can be easily measured or derived, as the chain length, the weight per unit length, or the equivalent hydrodynamic diameter; the accurate determination of the anchor position is a difficult step due to the difficulties of measuring and placing it under the water at the exact specified distance of several meters. For this reason, looking at the static shape measured by the underwater cameras, we concluded that the exact anchor position measured from the basin wall was $5.305 \mathrm{~m}$ (See Figure 3). The results, in particular 
the dynamic tension, are sensitive to small variations of the anchor position. Assuming this anchor position, the agreement of the computational and the experimental static shape of the chain is excellent in both configurations, as it is shown in Figure 4 .

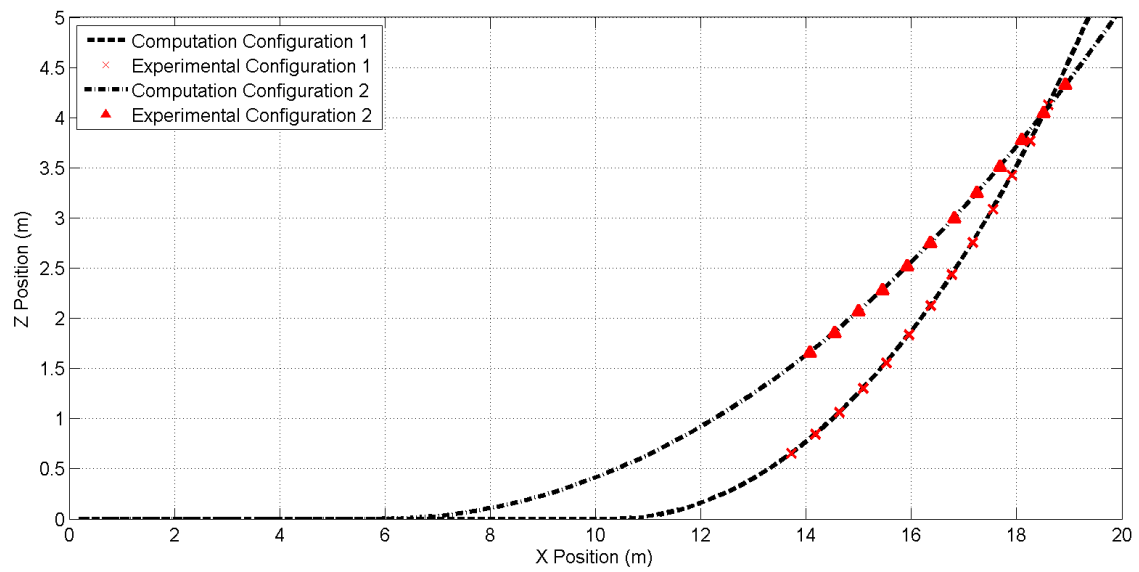

Figure 4: Comparison of the computed and experimental static shapes for both line configurations

Table 5 shows the difference between the computed tension at the fairlead and the experimental measurement for both configurations. The error in the predicted static tension is below $2 \%$.

Table 5: Comparison of static fairlead tension expressed in Newtons.

\begin{tabular}{lcc}
\hline \hline & Configuration 1 & Configuration 2 \\
\hline Experimental & 8.13 & 14.48 \\
Computation & 8.10 & 14.70 \\
\hline Difference & $0.37 \%$ & $1.52 \%$ \\
\hline
\end{tabular}




\subsection{Dynamic Cases: Tension at the Fairlead}

Figures 5 and 6 show the comparison between the numerical predictions and the experiments for the different periods of the motion prescribed at the fairlead of the chain for Configuration 1 and Configuration 2, respectively. The plots show the fairlead tension of the chain against the fairlead position. The initial transients have been eliminated and the data is plotted once the steady state has been reached. The arrows represent the sense of the dynamic loop: higher tensions are achieved when the fairlead of the chain is moving away from the anchor and the lower tensions appear when it is approaching the anchor.

For the experimental data, the loops have been generated from time series containing several tens of periods of the prescribed motion. As the measured signal presents a certain amount of noise, instead of plotting the direct out-coming data, the mean value and the standard deviation of the data have been computed. These statistical data is represented in the graphs instead of the raw data for clarity. The mean value is represented by a circle and the standard deviation by a vertical bar centered at the mean and limited by horizontal lines. The definition of the standard deviation, $\sigma$, is given in Equation (15):

$$
\sigma=\sqrt{\frac{\sum_{i=1}^{N}\left(X_{i}-\bar{X}\right)^{2}}{N}},
$$

Where $N$ is the number of elements in the population, $\bar{X}$ is the population mean and $X_{i}$ is the $i^{\text {th }}$ element of the population.

In addition, the plots show the fairlead tension of the chain for the initial static position (red circle) and also the tension provided by the Quasi-Static model for the different positions of the fairlead (gray line). 


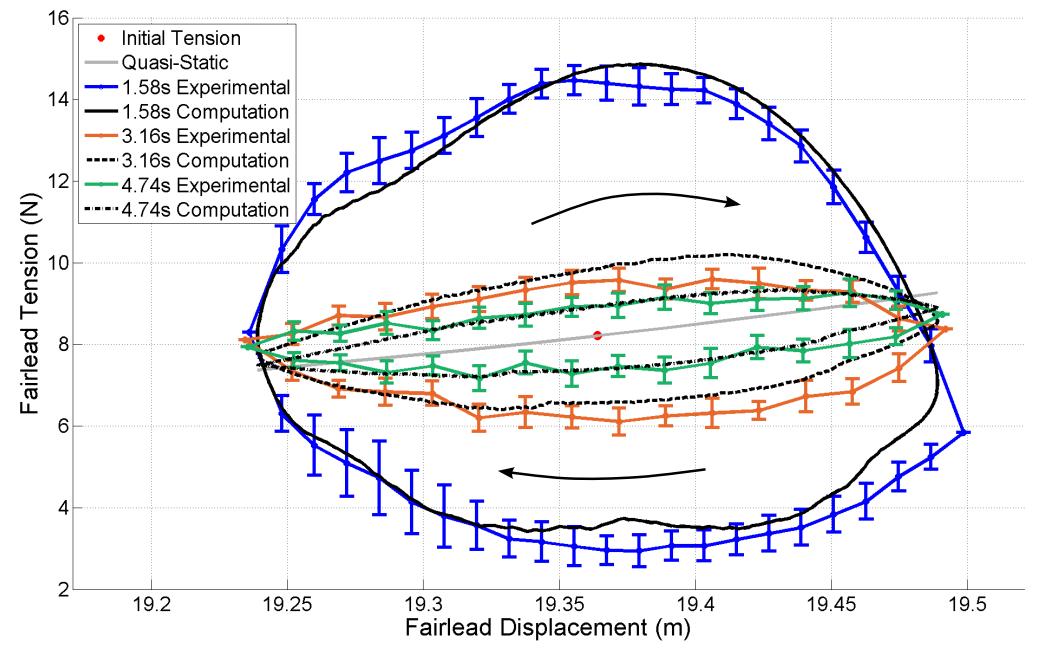

Figure 5: Fairlead tension for the different periods used in the dynamic simulations for Configuration 1. The tension at the fairlead for the initial static position (red circle) and also the tension provided by the Quasi-Static model for the different positions of the fairlead (gray line) are also represented. 


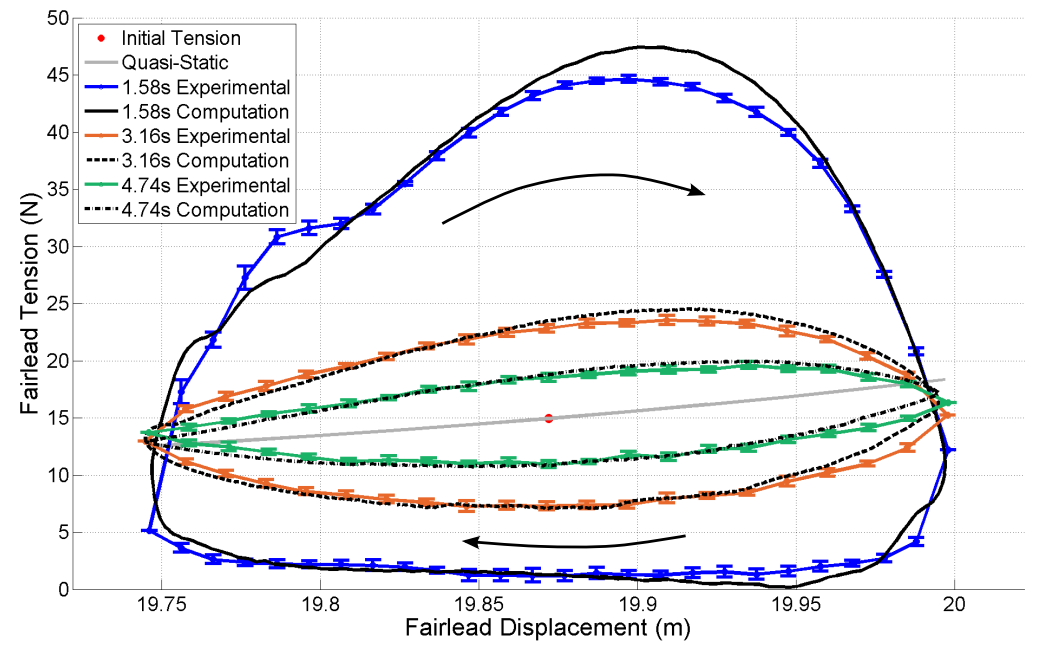

Figure 6: Fairlead tension for the different periods used in the dynamic simulations for Configuration 2. The tension at the fairlead for the initial static position (red circle) and also the tension provided by the Quasi-Static model for the different positions of the fairlead (gray line) are also represented.

As can be observed in Figures 5 and 6 , the tension in the Configuration 1 is lower than in the Configuration 2. In fact, the initial static tension for Configuration 1 is $8 \mathrm{~N}$ and for Configuration 2 it is $15 \mathrm{~N}$, and the maximum tension, for the $1.58 \mathrm{~s}$ period, in Configuration 2 is three times the value in Configuration 1. As has been mentioned, the tension signal acquired during the tests, presents a certain amount of noise. This is the reason why the relative importance of the tension standard deviation in Configuration 1 is higher than in the second one. The figures also illustrate the importance of dynamic effects, in particular as the excitation frequency increases. The maximum tension for the dynamic computation of the case with $1.58 \mathrm{~s}$ of excitation period can be between 2 (Configuration 1) and 3 (Configuration 2) times the value predicted by the Quasi-Static approach. For fatigue calculations of the fairlead and line, the dynamic effects are even more significant. For Configuration 2 with $1.58 \mathrm{~s}$ excitation period, the dynamic tension amplitude is more than ten times times 
the corresponding quasi-static value.

The agreement between computations and measured data is, in general, very good for both configurations and all the excitation periods. The two highest excitation periods, $3.16 \mathrm{~s}$ and $4.74 \mathrm{~s}$, correspond to the chain's Harmonic Condition described in [17]. In these cases the agreement is good, in particular for Configuration 2, where the relative importance of the noise, and the standard deviation of the data, is lower.

For the lowest period of excitation, $1.58 \mathrm{~s}$, the dynamics of the chain correspond to the Snap Condition which is more complex. In Configuration 1 the chain partially loses tension, and in Configuration 2 the situation is more extreme and the chain totally loses tension; the measured tension at the fairlead is 0 , during almost half of the period. Nevertheless, the agreement with computa250 tions is still good and a discrepancy of around $4.5 \%$ on the estimation of the maximum tension appears in both configurations. This maximum tension appears approximately when the velocity of the chain 's fairlead is maximum. As described in Section 6, we have chosen the tangential and normal drag coefficients for the computations based on the values provided by the guidelines. These values provide an accurate matching with experimental results, though further research on the characterization of these parameters for the regime with highest excitation frequency may result on a better prediction of the maximum tension for the $1.58 \mathrm{~s}$ period case.

Another slight discrepancy appears in both configurations for the period of $1.58 \mathrm{~s}$. When the fairlead moves towards the anchor, the chain loses tension. The fairlead motion reverses, the tension is recovered, and the links that are falling freely suddenly are pulled upwards, producing a snap load; a sudden increase of the tension. During this event, the measured tension temporarily reaches a plateau, before again increasing towards the peak value. For the experimental 265 data this decrease in the slope appears later and it is more pronounced than in the computational results. This effect is even more important in the Configuration 2 case.

The case for Configuration 2 and oscillation period of $1.58 \mathrm{~s}$, where the loss of 
tension of the line is the most pronounced, is very sensitive to the modelling of the seabed-cable interaction. For this reason, this case was studied more in detail and was simulated with the alternative code 3DFloat, developed by IFE. 3DFloat [3] is based on a nonlinear co-rotated FEM approach, with three translational and three rotational DOFs at each node. The cable elements are Euler-Bernoulli beams, where the bending stiffness is turned off for chain mooring lines. Both codes have elements that maintain axial stiffness also when tension is lost, in contrast to the real chain. This can affect the behaviour for the part of the chain resting on the seafloor. Both models take the seafloor into account with a seabed contact model. Nodes that drop below the defined seafloor, get an applied vertical force that is proportional to the vertical distance to the seafloor. In addition, linear damping is applied to nodes below the seafloor in the vertical direction. Both codes scale the linear damping with the distance up to the seafloor. 3DFloat also applies a limiter to the damping. 3DFloat uses no friction for the computations in this paper, but applies a horizontal damping to the nodes in contact with the seabed, that is not present in OPASS

Figure 7 shows the effect of eliminating the seabed-cable friction on the tension computed with OPASS for the mentioned sensitive case of period $1.58 \mathrm{~s}$ and Configuration 2. After a few periods of excitation, an oscillation is generated in the part of the loop where the suspension point moves from the minimum position towards the maximum position and the chain is being lifted quickly off the seafloor. This effect does not arise in any of the other cases studied in this work when the seabed friction model is disabled.

When the considered case is simulated with 3DFloat, a similar effect arise (see Figure 8); if no damping is applied to the nodes in contact with the seabed, an oscillation appears when the line is recovering the tension. But if enough damping is applied in the vertical and horizontal direction to the nodes in contact with the seabed, this dynamics disappears.

Apparently, these dynamics are caused by small motions of the nodes of the line resting on the seabed during the tension-less portion of the dynamic loop. The 
introduction of seabed friction in the OPASS model or seabed damping in the 3DFloat model avoid these small nodal displacements and, consequently, the oscillations are not generated. These dynamics are not present in the experimental results, due to the friction of the chain with the bottom of the basin. This case highlights the importance of a correct modelling of the line-seabed interaction to avoid the generation of instabilities in highly dynamic situations with pronounced loss of tension in the chain.

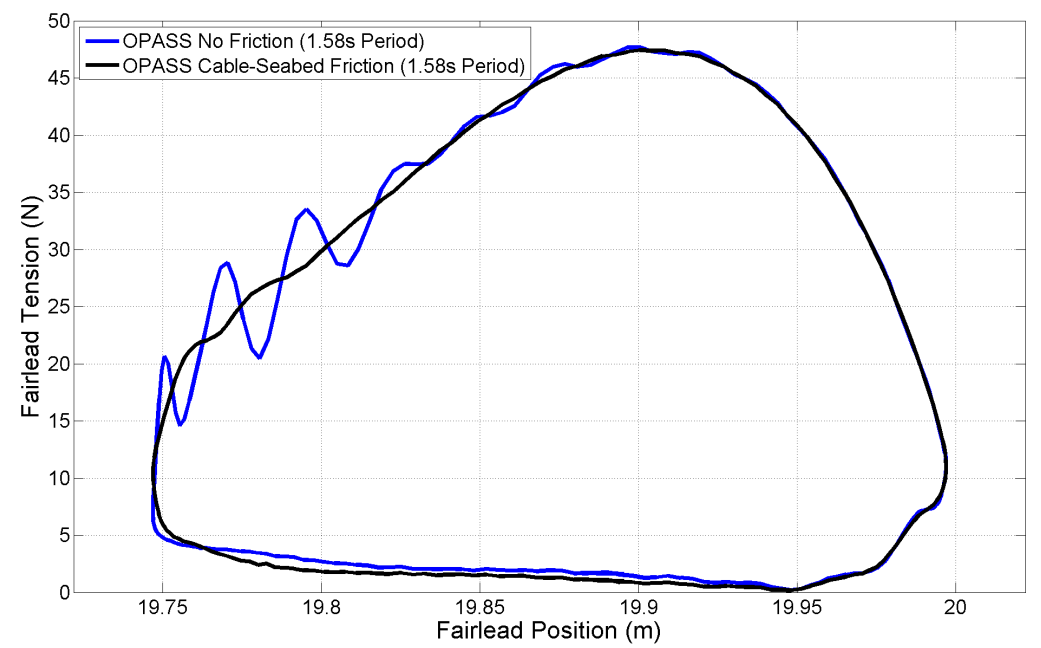

Figure 7: Effect of seabed friction on the fairlead tension computed with OPASS for $1.58 \mathrm{~s}$ period and Configuration 2 


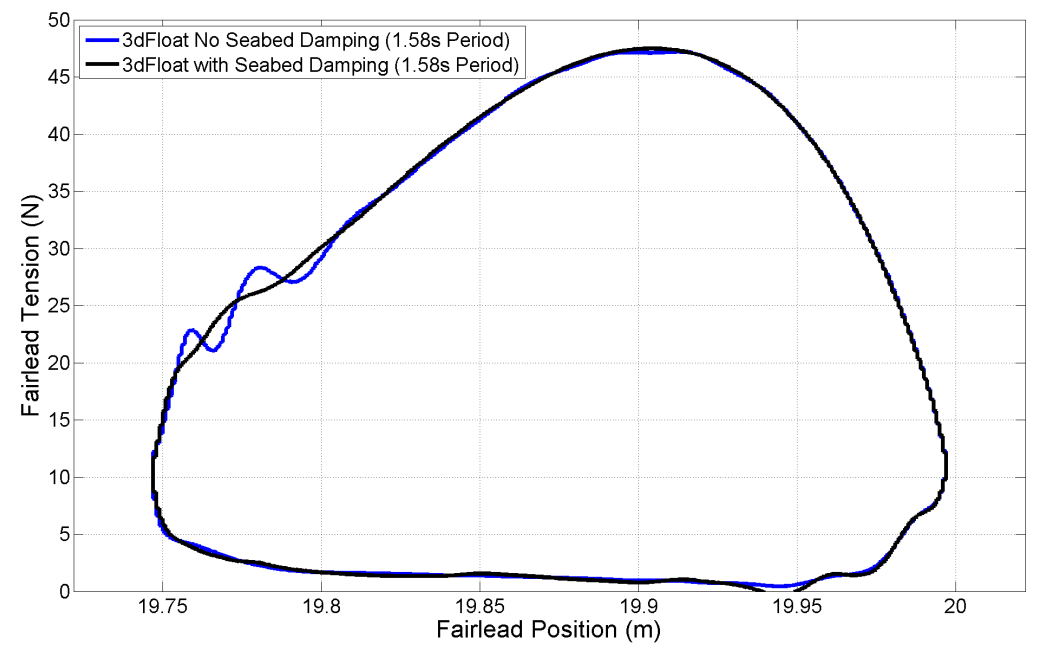

Figure 8: Effect of seabed damping on the fairlead tension computed with 3DFloat for 1.58 s period and Configuration 2

Figure 9 shows how the two models agree well for the $1.58 \mathrm{~s}$ period case, considering the modelling differences, once the friction is included in OPASS and the seabed damping is included in 3DFloat. The results are not sensitive to further increases of friction or damping levels beyond damping the oscillations seen in the Figure 7 and Figure 8 . For the larger periods the results are not sensitive to the seabed model and both tools provide almost identical tension. 


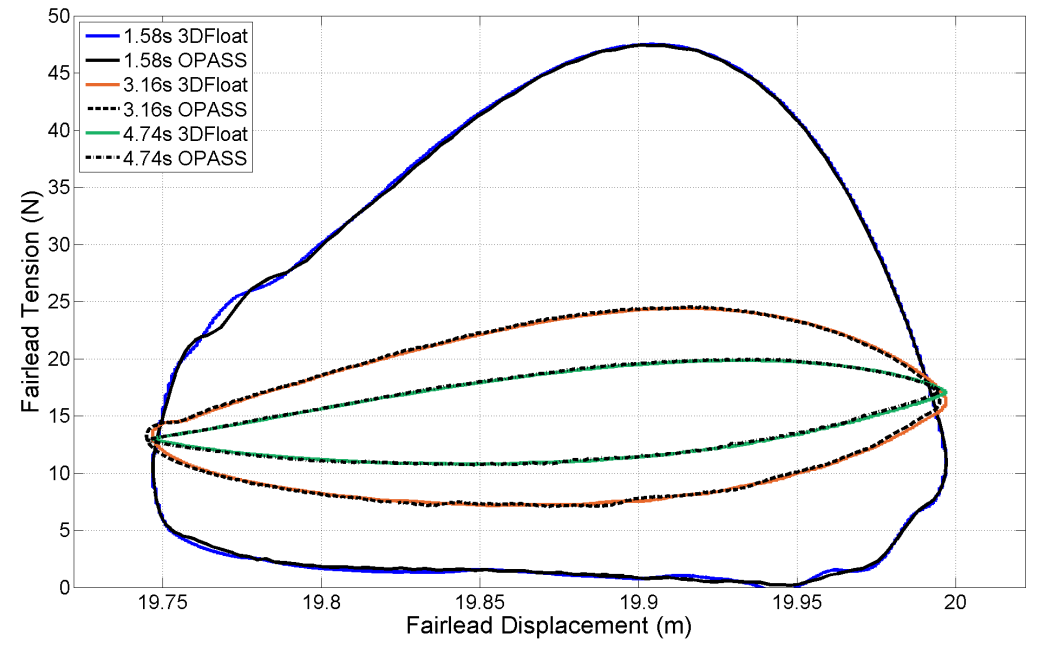

Figure 9: Comparison of 3DFloat and OPASS tension results for Configuration 2

In summary, the computational model is able to predict with good precision

the tension in all the cases considered. In those cases where chain loses tension and the dynamics is more complex, the computations also predict the tension with accuracy, though special attention has to be paid to the seabed-cable interaction model.

\subsection{Dynamic Cases: Motion of the Chain}

A comparison between the measured and the computed trajectories of the 8 positions along the chain length specified in Table 2 is discussed in this section. Figures 10 to 25 show the trajectories of the markers for both configurations and for the different periods of excitation of the chain fairlead. According to the reference system described in Figure 3, the $x$ and $z$ coordinates represent the horizontal and vertical positions of the marker respectively.

Similarly to the procedure conducted in Section 7.2, the loops of experimental data have been generated based on several tens of periods of the prescribed motion and graphically represented by the mean value and the standard deviation. 
The dispersion of data for the motion is lower than in the tension results. For the calculation of the mean value and standard deviation, data has been stored into bins. The classification of data in these bins has been performed in most of cases based on the $x$ axis, but in some particular cases, as for example the motion of markers 5 and 6 for Configuration 2 and period 1.58s, see (Figures 19 and 21), the discretization is based in the $z$ axis, due to the shape of the curve and the requirements of the algorithm used in the data processing. In those cases, the dispersion bars on the plots are shown with their corresponding direction.

As before, the red dot in the plots show the initial static position of the marker before the chain is excited and the gray line represents the displacement of the 340 marker according to the Quasi-Static model. The arrows indicate the sense of the dynamic loops.

The scale and length of both the $x$ and $z$ axis is the same in all the plots from Figure 10 to Figure 25 to keep a constant reference frame for the chain at each marker position.

${ }_{345}$ The loop corresponding to period 1.58s represented in Figure 11 is not complete due to the loss of visibility of the marker by the tracking system during part of the cycle. 


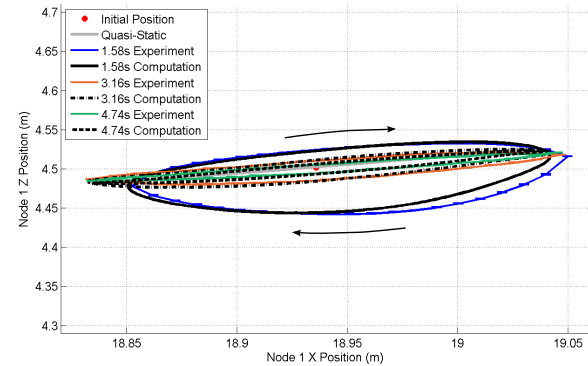

Figure 10: Trajectory described by the marker 1 (Conf. 1)

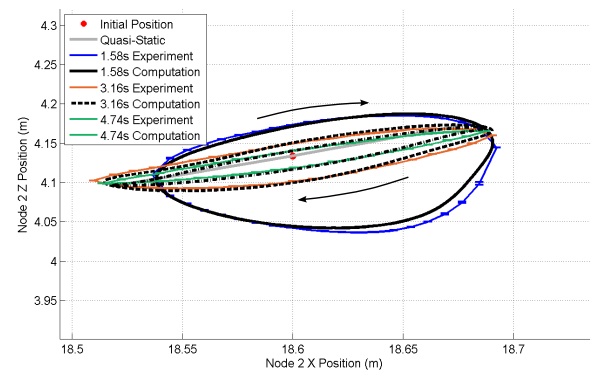

Figure 12: Trajectory described by the marker 2 (Conf. 1)

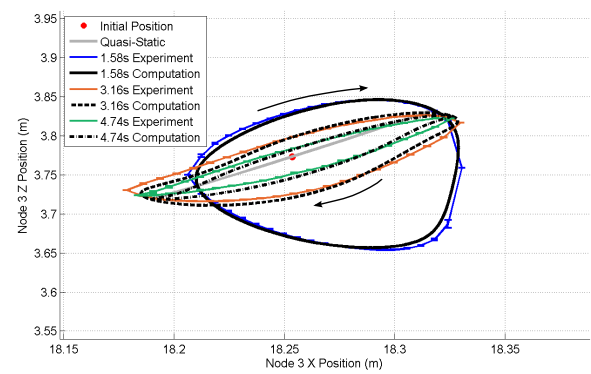

Figure 14: Trajectory described by the marker 3 (Conf. 1)

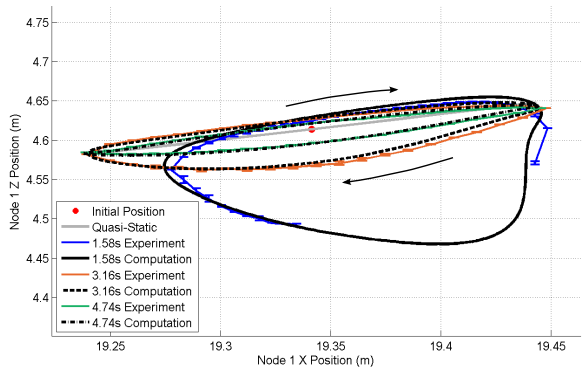

Figure 11: Trajectory described by the marker 1 (Conf. 2)

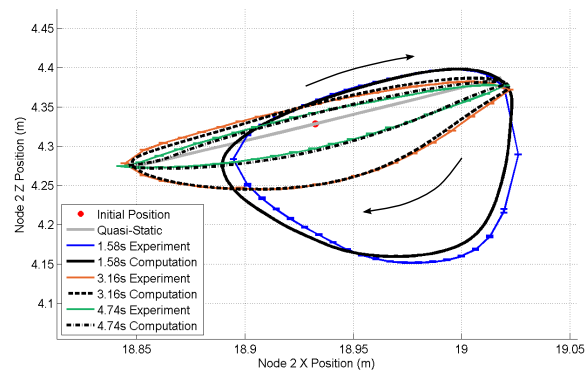

Figure 13: Trajectory described by the marker 2 (Conf. 2)

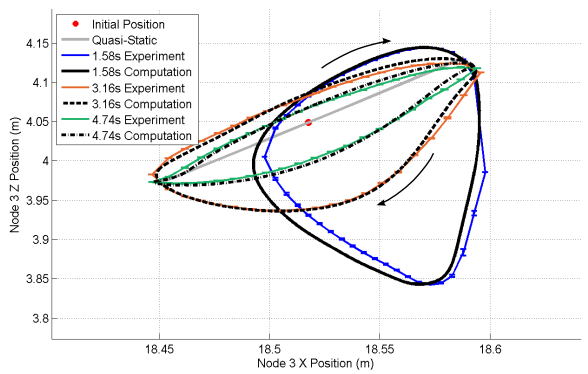

Figure 15: Trajectory described by the marker 3 (Conf. 2) 


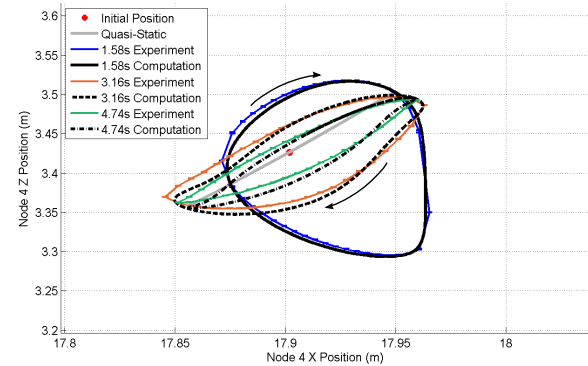

Figure 16: Trajectory described by the marker 4 (Conf. 1)

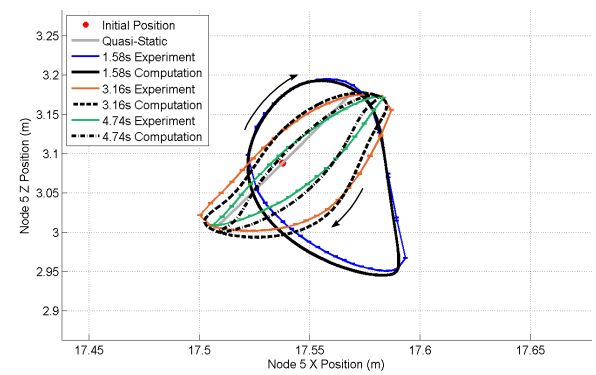

Figure 18: Trajectory described by the marker 5 (Conf. 1)

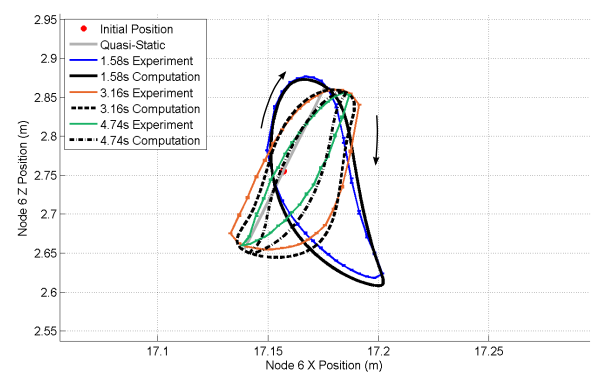

Figure 20: Trajectory described by the marker 6 (Conf. 1)

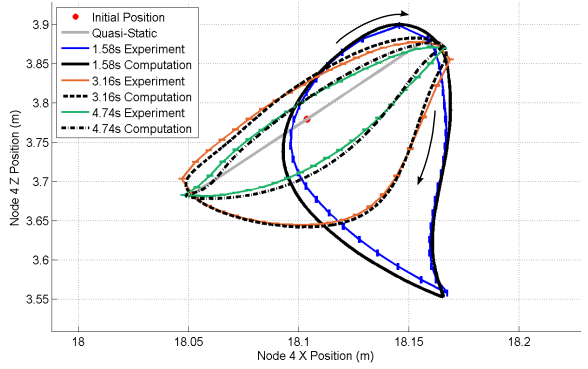

Figure 17: Trajectory described by the marker 4 (Conf. 2)

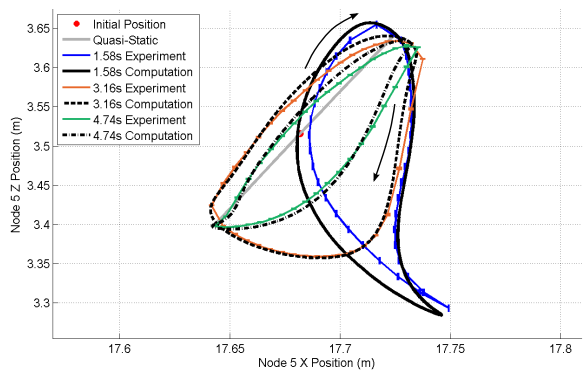

Figure 19: Trajectory described by the marker 5 (Conf. 2)

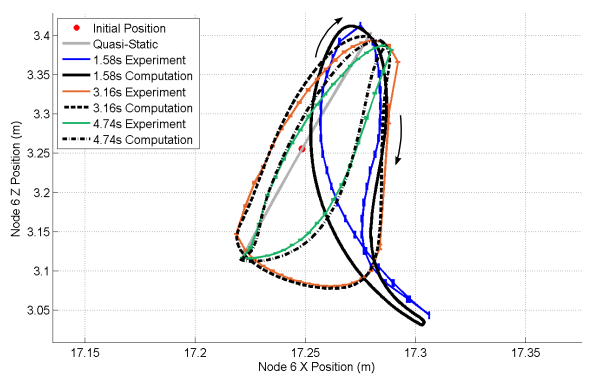

Figure 21: Trajectory described by the marker 6 (Conf. 2) 


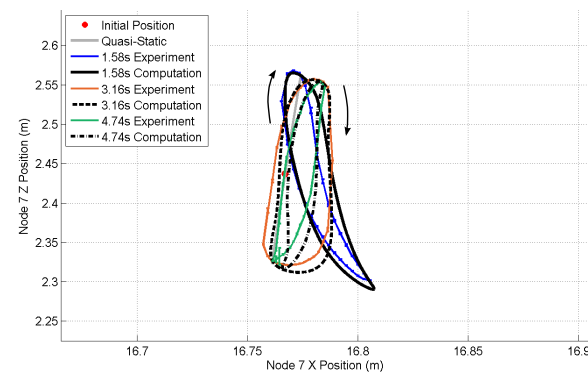

Figure 22: Trajectory described by the marker 7 (Conf. 1)

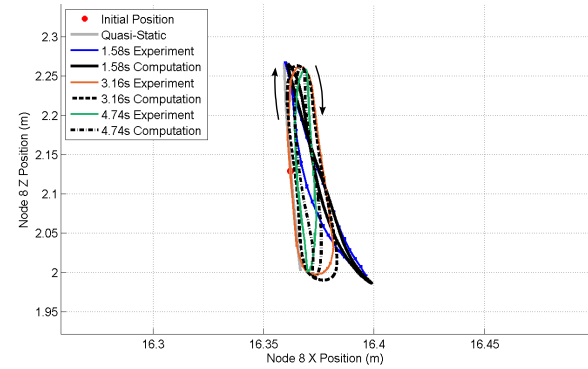

Figure 24: Trajectory described by the marker 8 (Conf. 1)

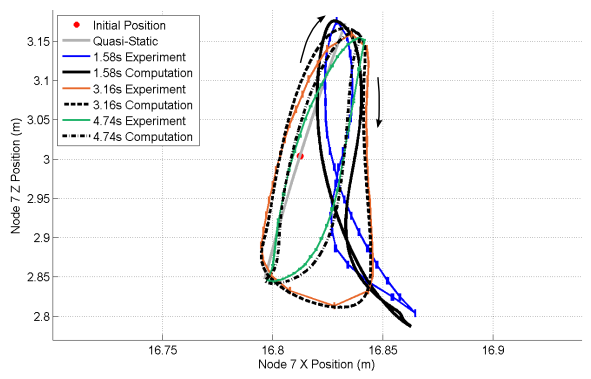

Figure 23: Trajectory described by the marker 7 (Conf. 2)

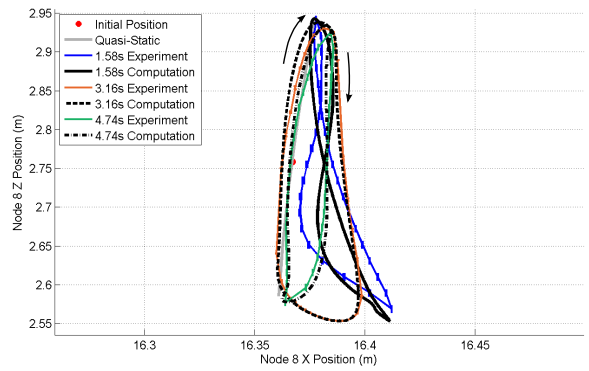

Figure 25: Trajectory described by the marker 8 (Conf. 2) 
In general, the agreement between the measured motions and the simulathe marker displacement in $x$ suffers a small oscillation giving a N-shape. This $\mathrm{N}$-shape effect is responsible for the secondary loops that appear at the deeper markers in the $x-z$ trajectories of Configuration 2 for period 1.58s, see Figures 
23 or 25. The N-shape is more pronounced as the distance along the chain to the fairlead increases. For this reason, no secondary loops are present at the markers closer to the fairlead, but they arise and increase their amplitude with the increasing distance to the excitation source. The dynamics of this effect on the loops is well predicted by the code, though it seems to be a complex effect and very sensitive to small variations in parameters. Thus, the trajectories of the markers located lower with the highest oscillation frequency (period 1.58s) and for Configuration 2 are the ones where the inaccuracy of the computations is higher, in particular for the $x$ coordinate.

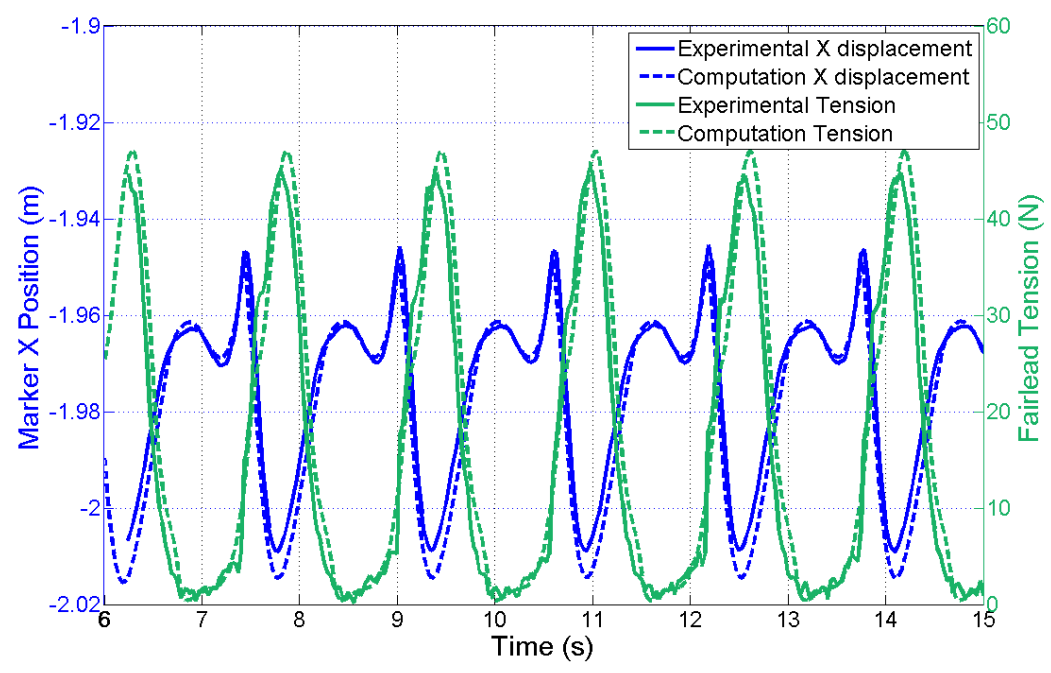

Figure 26: X position and tension for marker 5 (Conf. 2, period 1.58s) 


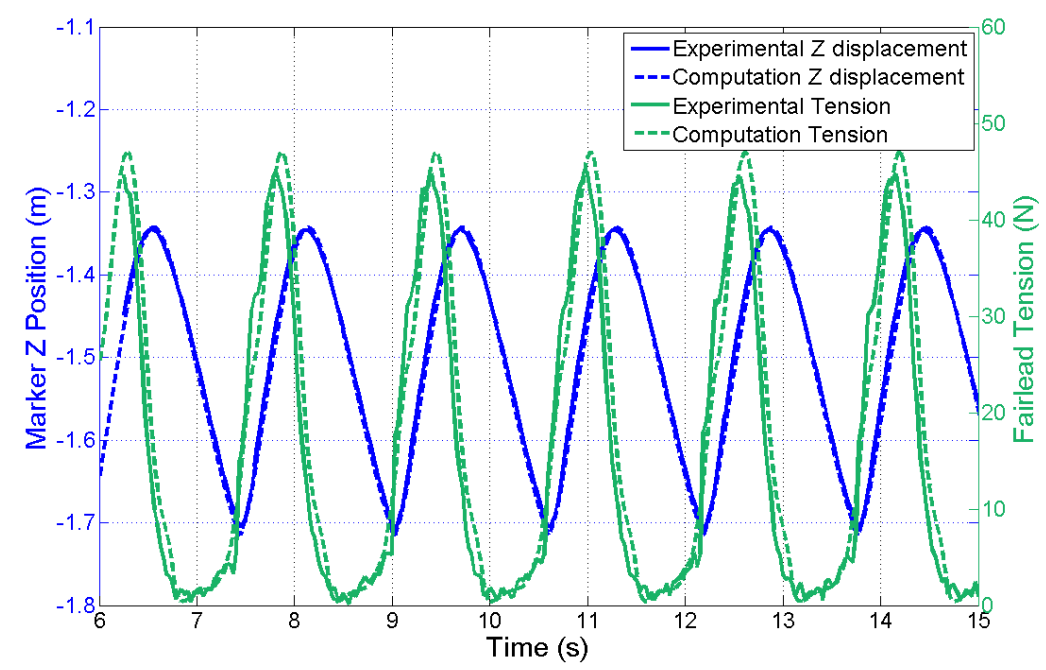

Figure 27: Z position and tension for marker 5 (Conf. 2, period 1.58s)

\section{Conclusions}

A new dynamic mooring lines code has been developed and successfully validated against experimental data for static and dynamic conditions. Two configurations of the chain have been studied, with different tension levels. The agreement between computations and experimental results is very good for the chain fairlead tension and also for the motion of the chain at the diverse positions considered.

In the static cases, both the shape of the line and the tension at the suspension point compare very well between the computations and the experiments.

For the dynamic validation, the chain has been excited with prescribed motion at the fairlead with three oscillation periods, producing different dynamic conditions, including harmonic response, loss of tension and snap loading. The code is able to predict the motion of the chain and the tension with precision in all these conditions. 
The added mass and drag coefficients for the chain that have been chosen from guidelines, in general, represent with accuracy the hydrodynamic loads.

The importance of including dynamic effects on the prediction of the mooring line loads has been shown; the maximum tension can between 2 and 3 times the value computed by the Quasi-Static approach when high excitation frequency is imposed. The tension amplitude can be more than ten times times the corresponding quasi-static value.

For the cases with the lowest excitation period, where the line slacks, the computed and measured tension agree well, although a slight difference appear during the snap loads once tension recovers. In these cases, the maximum tension is overpredicted by the code by around $4.5 \%$. These differences could be due to the fact that the drag coefficients were not tuned for the cases with the highest excitation frequency. In the chain motion prediction study, those cases where the chain loses tension present the highest inaccuracies in the numerical results, especially in the lowest part of the chain, though the results are still good.

In the cases with higher tension level and higher excitation frequency, the simulation results are particularly sensitive to the cable-seabed interaction model. This was confirmed also by verification against the 3DFloat code of IFE. The computational model is able to predict the tension with accuracy, but the modelling of the seabed friction or the seabed damping should not be neglected.

\section{Acknowledgments}

We would like to thank Jean Marc Rousset, Sylvain Bourdier and Laurent Davoust from ECN, for their assistance and experienced work during the execution of the tests at Nantes. The testing leading to these results has received support from MARINET, a European Community-Research Infraestructure Action under the FP7 "Capacities" Specific Programme.

The major part of this work has been funded by the European Community's FP7 INNWIND.EU project, under grant agreement number 308974. The Spa- 
nish Ministry for Science and Innovation has also funded the work under grant TRA2013-41096-P "Optimization of liquid gas transport for LNG vessels by fluid structure interaction studies". Finally, the authors would like to acknowledge support from the Norwegian Research Centre for Offshore Wind Technology (NOWITECH).

\section{References}

\section{References}

[1] Azcona J., Munduate X., Nygaard T. A. and Merino D., 2011. Development of OPASS Code for Dynamic Simulation of Mooring Lines in Contact with Seabed, in: EWEA Offshore 2011, Amsterdam, The Netherlands.

[2] Bureau Veritas, 2002. NR 493 R02 E, Classification of Mooring Systems for Permanent Offshore Units.

[3] De Vaal J. B. and Nygaard T. A., 2015. 3DFloat User Manual. Report IFE/KR/E-2015-001. Institute for Energy Technology, Norway

[4] DNV, 2013. DNV OS E301 Position Mooring - DNV offshore Standards Det Norske Veritas, Høvik, Norway.

[5] Hall M., Goupee A., 2015. Validation of a Lumped-Mass Mooring Line Model with OC4-DeepCwind Semisumersible Model Test Data. Ocean Engineering, Vol 104, pp. 590-603. doi: 10.1016/j.oceaneng.2015.05.035.

[6] Hansen, M. O. L., 2008. Aerodynamics of Wind Turbines. Earthscan, London, England.

[7] Jonkman J. M., 2007. Dynamics Modeling and Loads Analysis of an Offshore Floating Wind Turbine. PhD Thesis, University of Colorado, USA.

[8] Masciola M., Robertson A., Jonkman, J. M. and Driscoll F., 2011. Investigation of a FAST-OrcaFlex Coupling Module for Integrating Turbine and 
Mooring Dynamics of Offshore Floating Wind Turbines, in: International Conference on Offshore Wind Energy and Ocean Energy, Beijing, China.

[9] Morison J. R., O'Brien, M. P., Johnson, J. W. and Schaaf, S. A., 1950. The Force Exerted by Surface Waves on Piles. Journal of Petroleum Technology, vol. 2, no 05, pp. 149-154.

[10] Nakajima, V., Motora, S. and Fujino, M., 1982. On the Dynamic Analysis of Multicomponent Mooring Lines, in: 14th Offshore Technology Conference, OTC-4309(1982), pp. 105-121, Houston, USA. doi: 10.4043/4309-MS

[11] OrcaFlex 9.8b User Manual. Orcina, 2014.

[12] Papazoglou, V. J., Mavrakos, S. A. and Triantafyllou, M. S., 1989. NonLinear Cable Response and Model Testing in Water. Journal of Sound and Vibration, vol 140, no 1, P. 103-115.

[13] Pattison J. H., 1974. Components of Force Generated by Harmonic Oscillations of Small-Scale Mooring Lines in Water. Report number: SPD 589-01, David W. Taylor Naval Ship Research and Development Center, Bethesda, MD, USA.

[14] Robertson A., Jonkman J. M., Masciola M., Song H., Goupee A., Coulling A. and Luan C., 2014. Definition of the Semisubmersible Floating System for Phase II of OC4. Technical Report TP-5000-60601, National Renewable Laboratory (NREL), Golden (CO), USA.

[15] Robertson A., Jonkman J. M., Vorpahl F., Wojciech P., Qvist J., Frøyd L., Chen X., Azcona J., Uzunoglu E., Guedes Soares C., Luan C., Yutong H., Pengcheng F., Yde A., Larsen T., Nichols J., Buils R., Lei L., Nygaard T. A., Manolas D., Heege A., Vatne S. R., Ormberg H., Duarte T., Godreau C., Hansen H. F., Nielsen A. W., Riber H., Le Cunff C., Beyer F., Yamaguchi A., Jung K. J., Shin H., Shi W., Park H., Alves M. and Guérinel M., 2014. Offshore Code Comparison Collaboration Continuation Within IEA Wind Task 30: Phase II Results Regarding a Floating Semisumersible Wind 
System, in: 33rd International Conference on Ocean, Offshore and Arctic Engineering, OMAE, San Francisco, USA.

[16] Simos, A.N. and Fujarra, A.L.C., 2004. Dynamic Tension on Mooring Lines. Comparison of Numerical and Experimental Results, in: 14th International Offshore and Polar Engineering Conference, Toulon, France.

[17] Suhara, T., Koterayama, W., Tasai, F., Hiyama, H ., Sao, K. and Watanabe, K., 1981. Dynamic Behaviour and Tension of Oscillating Mooring Chain, in: 13th Offshore Technology Conference, OTC-4053(1981), pp. 415-424, Houston, USA. doi: 10.4043/4053-MS

[18] van den Boom, H.J.J., 1985. Dynamic Behaviour of Mooring Lines, in: BOSS Conference, Delft, The Netherlands.

[19] Walton, T. S. and Polacheck, H., 1960. Calculation of Non-Linear Transient Motion of Cables. Mathematics of Computation, 14, pp. 27-46.

[20] Wilhelmy, V., Fjeld, S. and Schneider, S., 1981. Non-Linear Response Analysis of Anchorage Systems for Compliant Deep Water Platforms, in: 13th Offshore Technology Conference, OTC-4051(1981), pp. 397-406, Houston, USA. doi: 10.4043/4051-MS 